\title{
CrystEngComm
}

Check for updates

Cite this: CrystEngComm, 2021, 23, 7108

Received 2nd July 2021,

Accepted 15th September 2021

DOI: $10.1039 /$ d1ce00878a

rsc.li/crystengcomm

\section{Isostructurality of quinoxaline crystal phases: the interplay of weak hydrogen bonds and halogen bonding $\dagger$}

\author{
Amie Saidykhan, Nathan W. Fenwick, (D) Richard D. Bowen, \\ Richard Telford and Colin C. Seaton (iD*
}

\begin{abstract}
Tailoring the physical properties of molecular crystals though the construction of solid solutions requires the existence of isostructural crystals. Simple substitutions of a given molecular framework can give a range of different crystal structures. A set of quinoxaline derivatives, $\mathrm{C}_{8} \mathrm{H}_{4} \mathrm{~N}_{2}\left(\mathrm{C}_{6} \mathrm{H}_{4} \mathrm{X}\right)_{2}, Q 3,3^{\prime} \mathrm{X}_{2}$, has been investigated ( $\mathrm{X}=\mathrm{F}, \mathrm{Cl}, \mathrm{Br}, \mathrm{I}$ and $\mathrm{Me}$ ) where kinetic factors generated a set of isostructural crystals for the lighter halogens $(\mathrm{F}, \mathrm{Cl}, \mathrm{Br})$ alone. Computational analysis shows that the stabilising interactions are maximal for $\mathrm{Cl}$, while DSC studies demonstrate the existence of more stable polymorphs for both $\mathrm{F}$ and $\mathrm{Br}$ containing systems. Steric factors appear to have a lower contribution than the balance of weaker hydrogen and halogen bonding shown by the $\mathrm{Me}$ and I containing systems displaying different packing driven by $\mathrm{CH} \cdots \mathrm{N} / \mathrm{CH} \cdots \pi$ bonds and $|\cdots|$ bonds respectively.
\end{abstract}

\section{Introduction}

A key objective of crystal engineering is the designed creation of functional materials with defined properties through control of the crystal packing. ${ }^{1}$ Crystal engineering has applications across many fields of science and engineering including adjusting pharmaceutical properties, ${ }^{2-5}$ electronic materials, ${ }^{6}$ energetic materials ${ }^{7,8}$ and optic/chromatic systems. ${ }^{9,10}$ Understanding how changes in molecular or compositional structure alters the final properties and potential crystal structures is thus a key topic for study. Isostructural systems, where different materials pack in similar crystal structures, offer a route to develop such understanding as the changes in physical properties with chemical alteration are directly correlated due to the consistency of crystal structure. The creation of solid solutions ${ }^{11,12}$ also requires isostructural pairs but offers the potential to vary systematically properties between two extremes. ${ }^{13}$ Predominately inorganic materials such as lead halide perovskites ${ }^{14}$ or metal-organic framework materials $^{15,16}$ are often are investigated through compositional changes within given crystal archetypes. This approach allows for controlled variation in the

School of Chemistry and Biosciences, University of Bradford, Bradford, Richmond Road, Bradford BD7 1DP, UK. E-mail: c.seaton@bradford.ac.uk

$\dagger$ Electronic supplementary information (ESI) available: Further experimental data (NMR, PXRD, DSC patterns of samples, crystallographic tables (pdf), crystal structures in cif format). CCDC 2093794-2093797. For ESI and crystallographic data in CIF or other electronic format see DOI: 10.1039/d1ce00878a physicochemical properties within a set crystal form by tailoring the level or choice of selected components. As such crystal structures are dominated by electrostatic forces between ionic components or the packing of complexes where the metal is hidden by the ligand structure such systematic changes are possible. In contrast, substitutional variation in organic molecular crystals often leads to significant changes in the crystal packing due to the changes in the array of weaker van der Waals and hydrogen bonding interactions that control the packing. There have been studies building from the initial work by Kitaigorodsky ${ }^{17}$ in the 1950 s into what factors influence the packing of organic crystals with studies into the replacement of $\mathrm{Cl}, \mathrm{Br}$ and I atoms, ${ }^{18-21} \mathrm{~F} / \mathrm{H}$ (ref. 22) or $\mathrm{CH}_{3} / \mathrm{Cl}$ replacement ${ }^{23}$ and the interchange of $\mathrm{C}=\mathrm{O}$ and $\mathrm{C}=\mathrm{S}$ groups ${ }^{24}$ Such studies highlight the role that similar molecular volumes and geometries play in controlling crystal packing. However, the inclusion of directional contacts such as halogen $\cdots$ halogen bonding ${ }^{25,26}$ and hydrogen bonding ${ }^{27}$ in the system can support or compete with geometric factors leading to challenges in identifying the directing factors for a given molecule. Thus, further creation and identification of new isostructural materials is required to achieve these outcomes. However, an additional challenge is often the difficulty in synthesising a full set of materials with the desired functional groups or substituents present or obtaining suitable crystals of each phase.

Quinoxaline derived molecules and polymers have shown a range of potential applications including biological activity $^{28}$ and optical properties. ${ }^{29}$ While this has led to significant numbers of papers developing efficient synthetic 
routes, few studies have investigated the impact of solid form choice (e.g. polymorphism) on physical properties. Examples include quinoxaline which is a dimorphic system with $Z^{\prime}=1$ and 5 forms, the latter formed by faster kinetic growth, ${ }^{30}$ $2(1 H)$-quinoxalinone $O$-(2'-quinoxalinyl)oxime which exist as yellow and orange polymorphs. ${ }^{31}$ The mechanofluorochromic material (E)-10-ethyl-3-(2-(3-methylquinoxalin-2-yl)vinyl)-10 $\mathrm{H}^{-}$ phenothiazine also has different coloured polymorphs (green and yellow) which display different light emission spectra. ${ }^{32}$ This demonstrates the potential versatility of such systems to form a range of polymorphic materials and tailoring of desired physical properties.

An experimental and computational study has been undertaken on the set $\mathbf{Q} 3 \mathbf{3}^{\prime} \mathbf{X}_{\mathbf{2}}$ (Scheme 1) where $\mathbf{X}=\mathrm{H}, \mathrm{F}, \mathrm{Cl}$, $\mathrm{Br}, \mathrm{I}, \mathrm{Me}$ to identify isostructural crystal structures and any relationships between the molecular, crystal structures and physical properties. These molecules offer a limited range of flexibility (Scheme 1) as steric clashes between the two aromatic rings would constrain the potential rotation of these groups. This restriction ensures that factors due to molecular geometry are limited across the substituent range. This should allow for identification of the influence of selected substituents upon the similarities and changes in the packing.

\section{Methodology}

\section{Experimental}

Synthetic procedure. The 2,3-diarylquinoxalines, Q3,3' $\mathbf{X}_{\mathbf{2}}$, ( $\mathrm{X}=\mathrm{H}, \mathrm{F}, \mathrm{Cl}, \mathrm{Br}$, I or $\mathrm{Me}$ ) were synthesised by condensation of 1,2-phenylenediamine, $\mathbf{P}$, and the requisite 2,2'disubstituted benzil, $\mathbf{B} 3,3^{\prime} \mathbf{X}_{2}$ in ethanol at $80{ }^{\circ} \mathbf{C} .{ }^{33}$ After addition of water to induce deposition of the crude product, careful recrystallisation from ethanol ( $\mathrm{X}=\mathrm{Cl}, \mathrm{Br}$ or $\mathrm{I}$ ) or ethanol and water $(\mathrm{X}=\mathrm{H}, \mathrm{F}$ or $\mathrm{Me}$ ) gave crystals suitable for analysis by crystallography. Typical yields were $45-70 \%$. In order to investigate the possibility that a different crystalline form might be formed from a non-polar solvent, a sample of each $\mathbf{Q} 3 \mathbf{3}^{\prime} \mathbf{X}_{\mathbf{2}}$ was recrystallised again from petroleum ether (60-80 ${ }^{\circ} \mathrm{C}$ fraction for $\mathrm{X}=\mathrm{H}, \mathrm{F}$ or $\mathrm{Cl}$; $80-100{ }^{\circ} \mathrm{C}$ fraction for $\mathrm{X}$ $=\mathrm{Br}$ or I).

Benzil ( $\mathrm{X}=\mathrm{H}$ ) was commercially available; four substituted benzils ( $\mathrm{X}=\mathrm{F}, \mathrm{Cl}, \mathrm{Br}$ or $\mathrm{Me}$ ) were obtained in 40$60 \%$ overall yield by the benzoin condensation ${ }^{34,35}$ of the appropriate 3-halogenobenzaldehyde, but with a thiazolium salt as catalyst instead of cyanide anion, followed by

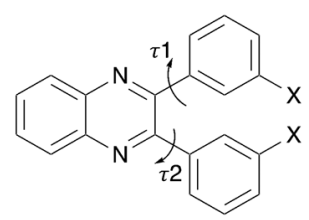

Scheme 1 Molecular structure of substituted quinoxaline $\left(Q 3,3^{\prime} X_{2}\right)$ where $\mathrm{X}=\mathrm{H}, \mathrm{F}, \mathrm{Cl}, \mathrm{Br}, \mathrm{I}$ or $\mathrm{Me}$. The two flexible torsion angles are indicated. oxidation of the resultant benzoin with nitric acid. ${ }^{33}$ The $3,3^{\prime}$ diiodobenzil was prepared in $50 \%$ yield by direct iodination of benzil with molecular iodine and sodium iodate in $90 \%$ sulphuric acid at $30{ }^{\circ} \mathrm{C} .{ }^{36}$ These synthetic routes are summarised in Scheme 2. All the intermediates and products were fully characterised by a combination of ${ }^{1} \mathrm{H}$ and ${ }^{13} \mathrm{C}$ nuclear magnetic resonance spectroscopy, infrared spectroscopy and mass spectrometry, as well as X-ray crystallography, and shown to be pure. Details of the synthesis and characterisation have been previous published. $^{37}$

Crystal structure determination. Single crystal X-ray diffraction data were collected on a Bruker X8 Apex II diffractometer using graphite monochromated Mo $\mathrm{K \alpha}$ radiation $(\lambda=0.7107 \AA)$ at $173 \mathrm{~K}$. The data were collected and reduced using Bruker SMART software. The structures were solved using intrinsic phasing in SHELXT $^{38}$ and refined by least squares in SHELXL ${ }^{39}$ within the olex2 package. ${ }^{40}$ Full crystallographic details are given in Table S1 in the electronic ESI. $\uparrow$ The resulting structures were deposited with the CCDC (deposition numbers: CCDC 2093794-2093797).

Powder X-ray diffraction. All powder X-ray diffraction data were collected using a Bruker D8 diffractometer with $\mathrm{Cu} \mathrm{K \alpha}$ radiation $(\lambda=1.5418 \AA)$ and a Lynxeye strip detector.

Thermal analysis. DSC data were collected using a TA instruments Q2000 equipped with a RCS90 cooling system. Samples (ca. $5 \mathrm{mg}$ ) were weighed accurately to T-zero aluminium pans and subjected to heat/cool/reheat cycles (reference pan of the same type) with heating and cooling rates of $10^{\circ} \mathrm{C} \mathrm{min}^{-1}$ between 20 to $210{ }^{\circ} \mathrm{C}$.

NMR analysis. Samples were prepared in deuterated chloroform $\left(\mathrm{CDCl}_{3}\right)$ at $\mathrm{ca} .10 \mathrm{mg} \mathrm{cm}{ }^{-3}$ before transferring 0.7 $\mathrm{cm}^{3}$ to precision $5 \mathrm{~mm}$ field matched NMR tubes (Wilmad, USA) and loading to a Bruker AVIII Neo $600 \mathrm{MHz}$ NMR spectrometer equipped with a $5 \mathrm{~mm}$ iProbe. ${ }^{1} \mathrm{H}$ spectra $(16$ scans) and NOESY spectra were acquired after optimising the

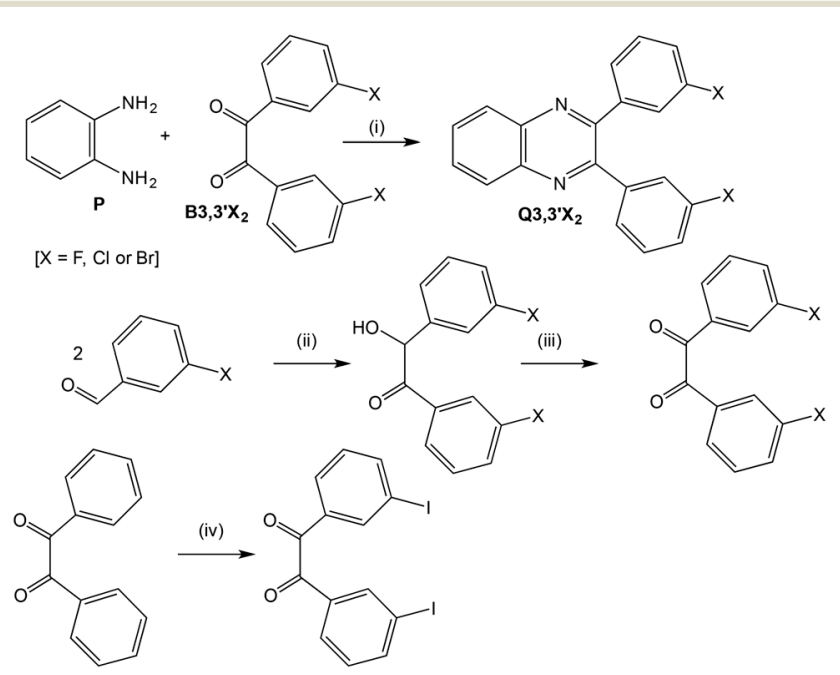

Scheme 2 Reagents and conditions: (i) $\mathrm{C}_{2} \mathrm{H}_{5} \mathrm{OH}$, reflux, 0.5-4 h; (ii) thiazolium salt, $\left(\mathrm{C}_{2} \mathrm{H}_{5}\right)_{3} \mathrm{~N}, \mathrm{C}_{2} \mathrm{H}_{5} \mathrm{OH}$, reflux, $2 \mathrm{~h}$; (iii) conc. $\mathrm{HNO}_{3}$, $\mathrm{CH}_{3} \mathrm{CO}_{2} \mathrm{H}, \mathrm{O}_{2}$, reflux, 2-4 h; (iv) $\mathrm{I}_{2}, \mathrm{NaIO}_{3}, 90 \% \mathrm{H}_{2} \mathrm{SO}_{4}, 30{ }^{\circ} \mathrm{C}, 1 \mathrm{~h}$. 
tune and match for the samples and shimming using Bruker's automated 'topshim' gradient shimming protocols. Processing of the spectra was carried out in TopSpin v. 4.0.8.

\section{Computational}

Conformational analysis. Molecular structures for each system were extracted from the crystal structures and the hydrogen location optimised in orca (V4.2.1) ${ }^{41}$ using the PBEh-3c DFT method. ${ }^{42}$ The conformations of each system were determined using a restricted systematic search, the desired torsion angle was held fixed in $5^{\circ}$ increments while the rest of the molecular was allowed to fully relax using the PBEh-3c level of theory. Each located minimum was then fully optimised to identify the lowest energy structures using the Grid5 and FinalGrid6 options.

Lattice energy and crystal energy frameworks. Lattice energies were evaluated using Materials Studio (COMPASSII forcefield) and in-house code (AA-CLP ${ }^{43}$ and $\mathrm{UNI}^{44}$ forcefields). The experimental structures were used as the starting point with hydrogen atom positions normalised. For COMPASSII calculations, atomic point charges were automatically assigned by the forcefield, while for the AA-CLP forcefield, atomic charges were fitted to the electron density evaluated at the PBEh-3c level in orca. For the in-house code the only positions of the molecules were optimised using the downhill simplex local optimisation algorithm, ${ }^{45}$ while the Materials Studio structures had positions and unit cell parameters optimised by local optimisation. Energy frameworks were determined using CrystalExplorer17 (ref. 46) with the intermolecular interactions evaluated using $\mathrm{HF} /$ 3-21G level for all systems. Dimers were then extracted from the crystal structures, hydrogen atomic positions were optimised using DFT PBEh-3c method, followed by single point evaluation using B2PLYP-D3/def2-QZVPP ${ }^{47}$ DFT calculations with the FinalGrid6 option in orca.

Crystal structure prediction. Crystal structure prediction was undertaken with the prediction module in Materials Studio, using the COMPASSII forcefield to evaluate the energy. The molecular structure was allowed to be freely rotated about the two torsion angles, during generation of structure.

\section{Results and discussion}

Single crystal analysis determined new crystal structures for $\mathbf{Q 3}^{\prime} \mathbf{3}^{\prime} \mathbf{X}_{\mathbf{2}}$ (X = F, Cl, I, Me, Table S1 $\dagger$ ), while it confirmed that the samples for $\mathrm{X}=\mathrm{H}$ and $\mathrm{Br}$ had the same structures as the previously determined crystals $(\mathrm{X}=\mathrm{H}$; REFCODE $=$ ODEJAJ, $\mathrm{X}$ $=\mathrm{Br}$; REFCODE $=$ EDESAI). While most of the bond lengths and angles are in the expected range, the steric factors of the substituted rings alter the bond lengths of the quinoxaline ring (Table 1).

For $\mathrm{X}=\mathrm{H}, \mathrm{F}, \mathrm{Cl}$ and $\mathrm{Br}$, the conformation in the crystal was the same, while for $\mathrm{X}=\mathrm{I}$ and $\mathrm{Me}$ have a common conformation that differs from the others (Table 2). No disorder or unusual vibrational features were observed in the ADPs for the systems (Fig. 1). To confirm whether the observed differences are due to the choice of functional group or a factor due to the crystal packing the flexibility of the conformation was investigated through computational methods.

A single torsion angle was systematically varied through $360^{\circ}$, while the rest of the molecule was allowed to relax freely. This plots out the lowest energy landscape for the molecular conformations. Four symmetry related minima were located for $\mathbf{Q 3}, \mathbf{3}^{\prime} \mathbf{H}_{\mathbf{2}}$ which lose their degeneracy upon substitution (Table 3). However, the various minima have very similar energies despite the differences in orientation of the functional groups or substituents (Fig. 2).

Given the low energies of the minima, it can be seen that suitable crystal packing forces would be able to compensate for the energy penalty packing in any of these minima. As the barriers to rotation are approximately $20 \mathrm{~kJ} \mathrm{~mol}^{-1}$ for all systems, slightly higher than in straight chain alkanes (approximately $16 \mathrm{~kJ} \mathrm{~mol}^{-1}$ ), ${ }^{48}$ interconversion of the different conformation should be possible. Solution ${ }^{1} \mathrm{H}$ NOESY NMR studies (Fig. S1†) show no evidence for the preferential formation of distinct conformations. Thus, the difference in conformation in the crystal structures appears to be driven by crystal packing rather than a molecular preference derived by the choice of functional group.

\section{Crystal structure analysis}

The packing of $\mathbf{Q 3}, \mathbf{3}^{\prime} \mathbf{H}_{\mathbf{2}}$ is dominated through $\mathrm{CH}^{\cdots} \pi$ interactions with a dimer formed by phenyl-to-phenyl

Table 1 Measured bond lengths in the pyrazine in $Q 3,3^{\prime} X_{2}$

\begin{tabular}{llr}
\hline $\mathrm{X}$ & $d_{\mathrm{C}-\mathrm{N}} / \AA$ & $d_{\mathrm{C}-\mathrm{C}} / \AA$ \\
\hline $\mathrm{H}$ & $1.368(2), 1.368(2), 1.321(2), 1.323(2)$ & $1.413(2), 1.445(2)$ \\
$\mathrm{F}$ & $1.371(2), 1.363(3), 1.314(3), 1.323(2)$ & $1.414(3), 1.442(2)$ \\
$\mathrm{Cl}$ & $1.369(3), 1.368(3), 1.320(3), 1.321(3)$ & $1.415(3), 1.443(3)$ \\
$\mathrm{Br}$ & $1.360(5), 1.360(6), 1.315(4), 1.309(5)$ & $1.390(6), 1.430(5)$ \\
$\mathrm{I}$ & $1.369(5), 1.366(4), 1.319(4), 1.315(5)$ & $1.421(5), 1.448(5)$ \\
$\mathrm{Me}$ & $1.368(3), 1.366(3), 1.318(3), 1.316(3)$ & $1.421(3), 1.453(3)$ \\
Quinoxaline (HEYJOK02 $\left.Z^{\prime}=5\right)$ & $1.366(6), 1.362(6), 1.304(6), 1.308(6)$ & $1.413(6), 1.408(7)$ \\
& $1.376(6), 1.378(6), 1.307(6), 1.318(6)$ & $1.424(6), 1.398(7)$ \\
& $1.375(6), 1.366(6), 1.300(6), 1.317(6)$ & $1.398(6), 1.405(7)$ \\
& $1.365(6), 1.371(6), 1.303(6), 1.313(6)$ & $1.415(6), 1.402(7)$ \\
& $1.369(6), 1.382(6), 1.311(6), 1.292(6)$ & $1.420(6), 1.400(7)$
\end{tabular}


Table 2 Measured torsion angles in the different $Q 3,3^{\prime} X_{2}$ structures

\begin{tabular}{lll}
\hline $\mathrm{X}$ & Torsion 1 $\left({ }^{\circ}\right)$ & Torsion 2 (o) \\
\hline $\mathrm{H}$ & $-35.7(2)$ & $123.3(1)$ \\
$\mathrm{F}$ & $-45.0(2)$ & $131.3(2)$ \\
$\mathrm{Cl}$ & $-45.8(3)$ & $132.6(2)$ \\
$\mathrm{Br}$ & $-44.1(6)$ & $132.3(4)$ \\
$\mathrm{I}$ & $-41.0(5)$ & $-49.4(4)$ \\
$\mathrm{Me}$ & $-40.4(3)$ & $-51.8(3)$
\end{tabular}
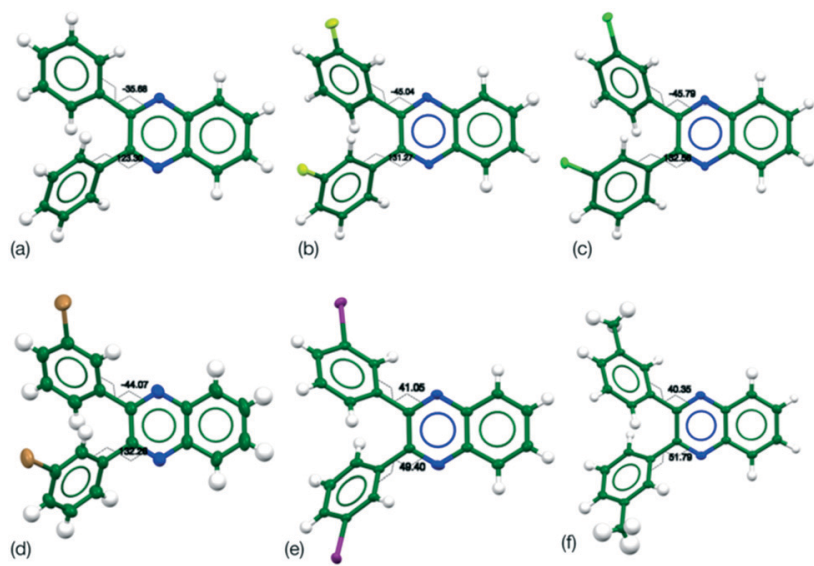

Fig. 1 Molecular conformations in the crystal structures of $Q 3,3^{\prime} X_{2}$ (a) $\mathrm{X}=\mathrm{H}$, (b) $\mathrm{X}=\mathrm{F}$, (c) $\mathrm{X}=\mathrm{Cl}$, (d) $\mathrm{X}=\mathrm{Br}$, (e) $\mathrm{X}=\mathrm{I}$, (f) $\mathrm{X}=\mathrm{Me}$. Carbon atoms are displayed as dark green spheres, nitrogen as blue, fluorine as yellow, chlorine as light green, bromine as brown, iodine as purple and hydrogen as white. Thermal ellipsoids are shown for 50\% probability levels for all.

interactions. Weak $\mathrm{CH} \cdots \mathrm{N}$ hydrogen bonds link these dimers into a 2-D sheet (Fig. 3). These sheets are stacked along the (101) direction through further $\mathrm{CH}^{\cdots} \pi \pi$ interactions. Until like substituted system there are no quinoxaline to quinoxaline $\pi$ interactions.

$\mathbf{Q 3}^{3} \mathbf{3}^{\prime} \mathbf{X}_{\mathbf{2}}$ (X = F, Cl, Br) crystals are isostructural (Table S1, Fig. S2 $\dagger$ ), all packing in a monoclinic $P 2{ }_{1} / n$ unit cell. The molecules are linked by $\mathrm{CH} \cdots \mathrm{N}$ hydrogen bonds into a 1-D spiral chain around the $2_{1}$ screw axis, which are then interlaced through $\pi \cdots \pi$ and $\mathrm{CH} \cdots \mathrm{Hal}$ interactions to form a 2-D layered structure in the $b c$-plane (Fig. 4). These layers then are stacked along the $a$-axis through further $\mathrm{CH} \cdots \mathrm{Hal}$ hydrogen bonds.
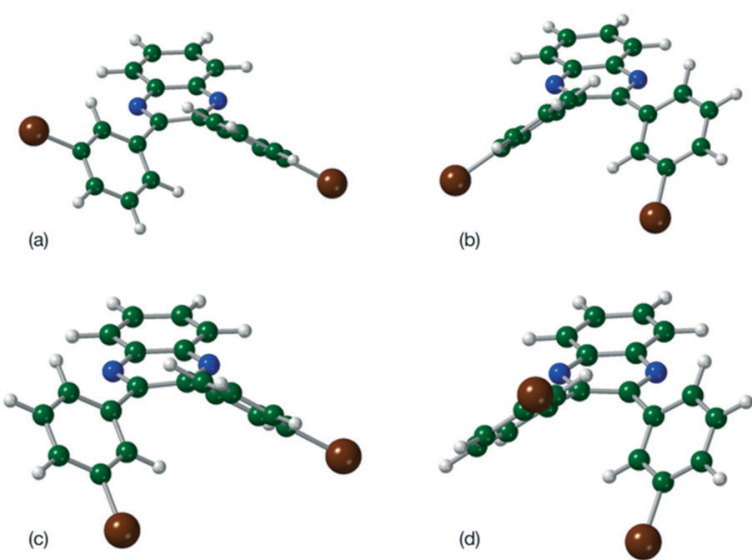

Fig. 2 Molecular geometries for the four located minima of $Q 3,3^{\prime} \mathrm{Br}_{2}$ (a) $\tau_{1}, \tau_{2}=-41^{\circ},-41^{\circ}$ (b) $\tau_{1}, \tau_{2}=41^{\circ},-136^{\circ}$ (c) $\tau_{1}, \tau_{2}=136^{\circ},-41^{\circ}$ and (d) $\tau_{1}, \tau_{2}=-136^{\circ}, 41^{\circ}$.

In $\mathbf{Q} 3, \mathbf{3}^{\prime} \mathbf{I}_{2} \quad \mathrm{CH}^{\cdots} \mathrm{N}$ hydrogen bonds form around the inversion centre dimerising pairs of molecules. Subsequent $\mathrm{CH} \cdots \mathrm{I}$ interactions generated by the $n$ glide plane link the dimers into a 2-D crinkled sheet. These sheets are then stacked along the $b$-axis by the $2_{1}$ screw axis with $\pi \cdots \pi$ and I $\cdots$ I interactions completing the crystal structure (Table 4) (Fig. 5).

In $\mathbf{Q 3}, \mathbf{3}^{\prime} \mathbf{M e}_{2}$, a $\mathbf{C H} \cdots \mathrm{N}$ hydrogen bond dimerises the molecules together. Another $\mathrm{CH} \cdots \mathrm{N}$ hydrogen bond forms a 1-D spiral ladder around the $2_{1}$ screw axis similarly to $\mathbf{Q 3}, \mathbf{3}^{\prime} \mathbf{F}$ / $\mathbf{C l} / \mathbf{B r}_{2}$, with further $\pi \cdots \pi$ interactions supporting the hydrogen bonds (Fig. 6, Table 4).

This analysis is supported by considering the energy frameworks of the crystal structures. These were calculated in CrystalExplorer at the $\mathrm{HF} / 3-21 \mathrm{G}$ level and show that $\mathbf{Q} 3, \mathbf{3}^{\prime} \mathbf{X}_{\mathbf{2}}$ (X $=\mathrm{F}, \mathrm{Cl}, \mathrm{Br}, \mathrm{I}$ ) form a hexagonal packed arrangement of strong interactions (Fig. 7) within a layered structure, although the location is varied with the changes in packing. The strongest interaction in each case corresponds to the identified 1-D chain in each system (Fig. 8) with the interaction strength increasing with substitution (Table 5).

Higher level theory calculations show good agreement for Q3,3' $\mathbf{H}_{2}$ but changes to halogen show increasing deviation. This may be due to the lack of dispersion factors in $\mathrm{HF}$ calculations and so an incorrect model of the halogen contacts in the dimers. However, the B2PLYP-D3 dimer energies suggest a drop in strength of the observed dimer for

Table 3 Relative energies of the four located conformational minima for $Q 3,3^{\prime} X_{2}$ calculated at DFT PBE3h-c level

\begin{tabular}{lllll}
\hline $\mathrm{X}$ & $\begin{array}{l}E_{1} / \mathrm{kJ} \mathrm{mol}^{-1} \\
\left(\tau_{1}, \tau_{2}=-41^{\circ},-41^{\circ}\right)\end{array}$ & $\begin{array}{l}E_{2} / \mathrm{kJ} \mathrm{mol}^{-1} \\
\left(\tau_{1}, \tau_{2}=41^{\circ},-136^{\circ}\right)\end{array}$ & $\begin{array}{l}E_{3} / \mathrm{kJ} \mathrm{mol}^{-1} \\
\left(\tau_{1}, \tau_{2}=136^{\circ},-41^{\circ}\right)\end{array}$ & $\begin{array}{l}E_{4} / \mathrm{kJ} \mathrm{mol}^{-1} \\
\left(\tau_{1}, \tau_{2}=-136^{\circ}, 41^{\circ}\right)\end{array}$ \\
\hline $\mathrm{H}$ & 0.01 & 0.01 & 0.00 & 0.00 \\
$\mathrm{~F}$ & 0.00 & 0.56 & 0.55 & 0.92 \\
$\mathrm{Cl}$ & 0.00 & 0.56 & 0.54 & 0.84 \\
$\mathrm{Br}$ & 0.00 & 0.59 & 0.57 & 0.92 \\
$\mathrm{I}$ & 0.00 & 0.39 & 0.00 & 0.52 \\
$\mathrm{Me}$ & 1.66 & 0.95 & & 0.91
\end{tabular}




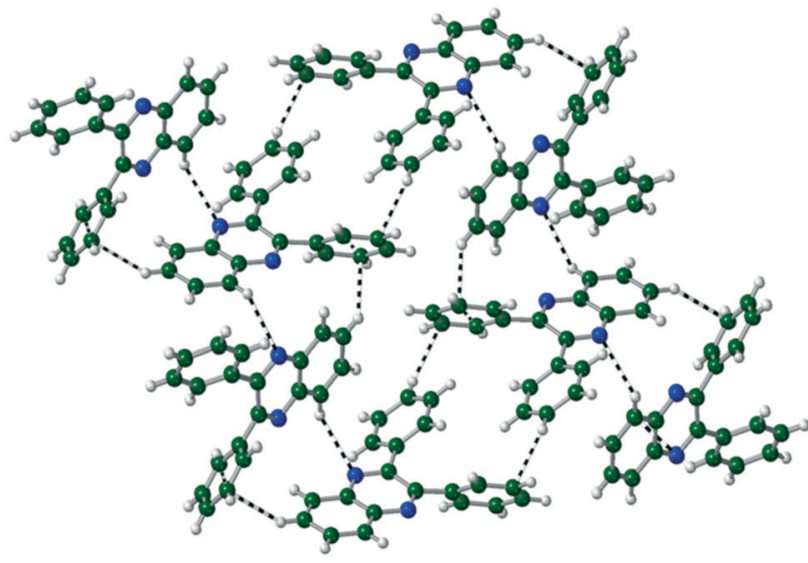

Fig. 3 Formation of 2-D sheet in $\mathrm{Q} 3,3^{\prime} \mathrm{H}_{2}$ through $\mathrm{CH} \cdots \mathrm{N}$ and $\mathrm{CH} \cdots \pi$ bonds. Viewed along the a-axis.

$\mathrm{Br}$ system. If such a drop continued for I, then resulting packing may be lower and so is the possible reason for a change in crystal packing. Calculation of the energy for a $\mathbf{Q} 3, \mathbf{3}^{\prime} \mathbf{F}_{2}$ dimer in the conformation observed in the $\mathbf{Q} \mathbf{3}, \mathbf{3}^{\prime} \mathbf{I}_{\mathbf{2}}$ structure shows a significant drop in interaction energy compared to the existing structure (B2PLYP-D3 $E=-18.22 \mathrm{~kJ}$ $\mathrm{mol}^{-1}$ ). This indicates that the choice of substitution is directing the choice of dimerization, thus the lattice energies for the known crystal structures were evaluated to identify whether these trends continued in the bulk energies from the dimers (Table 6). These show a general increase in lattice energy with substitution. In the case of COMPASSII and AACLP these forcefields use a single point electrostatic model which may inadequately model the halogen interaction, ${ }^{49}$ as is shown by very short I to I contacts in the optimised AACLP $\mathbf{Q} 3,3^{\prime} \mathbf{I}_{2}$ structure. In contrast, the UNI forcefield does not include electrostatic contributions and gives a better reflection of the B2PYLP-D3 results. These results indicate

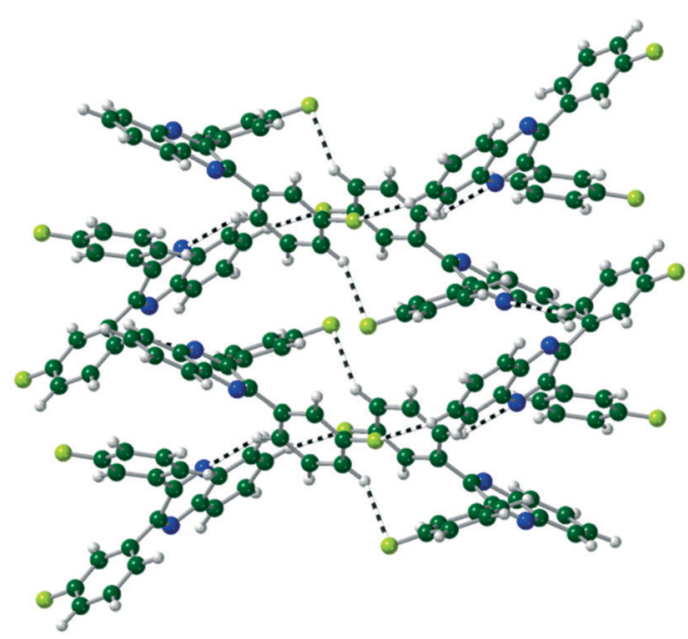

Fig. 4 Two interlocked spiral ladders formed through $\mathrm{CH} \cdots \mathrm{N}, \mathrm{CH} \cdots \mathrm{F}$ hydrogen bonds in the crystal structure of $Q 3,3^{\prime} F_{2}$. Viewed along the a-axis of the crystal.
Table 4 Selected weaker intermolecular interactions for $Q 3,3^{\prime} X_{2}$

\begin{tabular}{llll}
\hline $\mathrm{X}$ & Interaction & $d / \AA$ & Angle/ \\
\hline $\mathrm{H}$ & $\mathrm{CH} \cdots \pi\left(\mathrm{R} 2^{!}\right)$ & $2.80(1)$ & 138 \\
$\mathrm{H}$ & $\mathrm{CH} \cdots \pi\left(\mathrm{R} 2^{\dagger}\right)$ & $2.77(1)$ & 33 \\
$\mathrm{~F}$ & $\pi \cdots \pi\left(\mathrm{R} 1 \cdots \mathrm{R} 1^{\S}\right)$ & $3.722(1)$ & 0 \\
$\mathrm{~F}$ & $\pi \cdots \pi\left(\mathrm{R} 2 \cdots \mathrm{R} 2^{\dagger}\right)$ & $4.281(1)$ & 0 \\
$\mathrm{Cl}$ & $\pi \cdots \pi\left(\mathrm{R} 1 \cdots \mathrm{R} 1^{\S}\right)$ & $3.743(1)$ & 0 \\
$\mathrm{Cl}$ & $\pi \cdots \pi\left(\mathrm{R} 2 \cdots \mathrm{R} 2^{\dagger}\right)$ & $4.665(2)$ & 0 \\
$\mathrm{Br}$ & $\pi \cdots \pi\left(\mathrm{R} 1 \cdots \mathrm{R} 1^{\S}\right)$ & $3.822(2)$ & 0 \\
$\mathrm{Br}$ & $\pi \cdots \pi\left(\mathrm{R} 2 \cdots \mathrm{R} 2^{\dagger}\right)$ & $4.903(2)$ & 0 \\
$\mathrm{I}$ & $\pi \cdots \pi\left(\mathrm{R} 1 \cdots \mathrm{R} 1^{\dagger}\right)$ & $4.054(2)$ & 0 \\
$\mathrm{I}$ & $\pi \cdots \pi\left(\mathrm{R} 2 \cdots \mathrm{R} 2^{\ddagger}\right)$ & $4.652(2)$ & 57 \\
$\mathrm{I}$ & $\mathrm{C}-\mathrm{I} \cdots \mathrm{I}^{ \pm \pm}$ & $3.938(1)$ & $169 / 94$ \\
$\mathrm{Me}$ & $\pi \cdots \pi\left(\mathrm{R} 1 \cdots \mathrm{R} 2^{ \pm \pm}\right)$ & $3.708(2)$ & 14 \\
$\mathrm{Me}$ & $\pi \cdots \pi\left(\mathrm{R} 1 \cdots \mathrm{R} 2^{\S \S}\right)$ & $4.003(2)$ & 14 \\
$\mathrm{Me}$ & $\mathrm{CH} \cdots \pi\left(\mathrm{R} 2^{\dagger}\right)$ & $2.80(3)$ & 44
\end{tabular}

R1 = Quinoxaline ring, R2 = substituted benzene ring. Symmetry operations: ! $(-x, 2-y,-z) ; \S(1-x,-y, 1-z) ; \dagger(-x, 1-y, 1-z) ; \pm(1$ $-x, 1-y, 1-z) ; \ddagger(-1+x, y, z) ; \pm \pm(1 / 2-x,-1 / 2+y, 3 / 2-z) ; \S \S(1 / 2-$ $x, 1 / 2+y, 3 / 2-z) ; \dagger(-1 / 2+x, 3 / 2-y ;-1 / 2+z)$.

that packings are similar for all these system as expected by the lack of strong directional hydrogen bonding in these systems. To confirm that these are the lowest energy packings for these molecules, hypothetical structures for $\mathbf{Q 3}^{3}, \mathbf{3}^{\prime} \mathbf{F}_{2}$ and

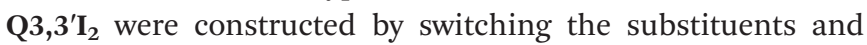
optimising the energies with Materials Studio and the COMPASSII forcefield. These generated structures were then also evaluated using AA-CLP and UNI forcefields (molecules position and orientations only optimised). In the case of Q3,3' $\mathbf{F}_{2}$ the crystal structures are of comparable energy to the observed structure, while $\mathbf{Q} \mathbf{3}, \mathbf{3}^{\prime} \mathbf{I}_{\mathbf{2}}$ favours the experimentally known structure. This suggests that it may be feasible to generate new polymorphs of $\mathbf{Q} \mathbf{3}, \mathbf{3 F}_{\mathbf{2}}$ with alternative conformations and crystal packings.

To explore the potential for other crystal structures, a short crystal structure prediction exercise was undertaken for $\mathbf{Q} 3, \mathbf{3}^{\prime} \mathbf{F}_{2}$ and $\mathbf{Q} 3, \mathbf{3}^{\prime} \mathbf{I}_{2}$. The resulting energy landscapes (Fig. 9)

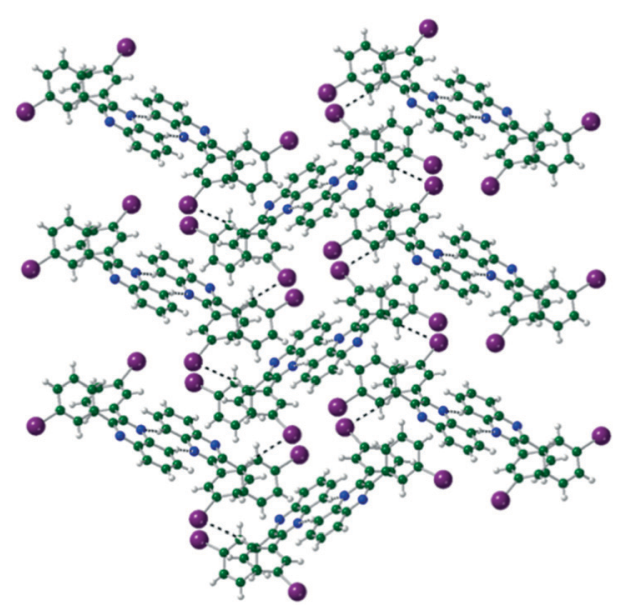

Fig. 5 Formation of sheet structure in $Q 3,\left.3^{\prime}\right|_{2}$. 


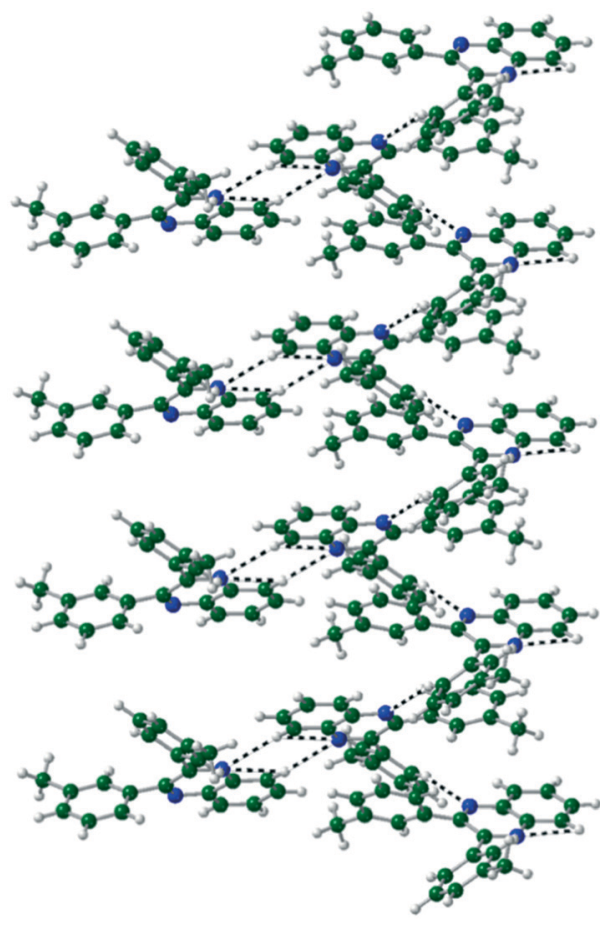

Fig. 6 Formation of spiral ladder linked through $\mathrm{CH} \cdots \mathrm{N}$ hydrogen bonds in $\mathrm{Q} 3,3^{\prime} \mathrm{Me}_{2}$.

show that there are significant numbers of feasible alternative structures for $\mathbf{Q} \mathbf{3}, \mathbf{3}^{\prime} \mathbf{F}_{\mathbf{2}}$ lower in energy than the experimentally obtained structure, but fewer for $\mathbf{Q 3}, \mathbf{3}^{\prime} \mathbf{I}_{\mathbf{2}}$. These structures display the range of potential low energy conformations suggesting that there is flexibility present in the system to allow a range of polymorphs to grow. In the Q3, $\mathbf{3}^{\prime} \mathbf{I}_{2}$ case, the same lowest energy conformation is present in the predicted structures as the experimental structure.
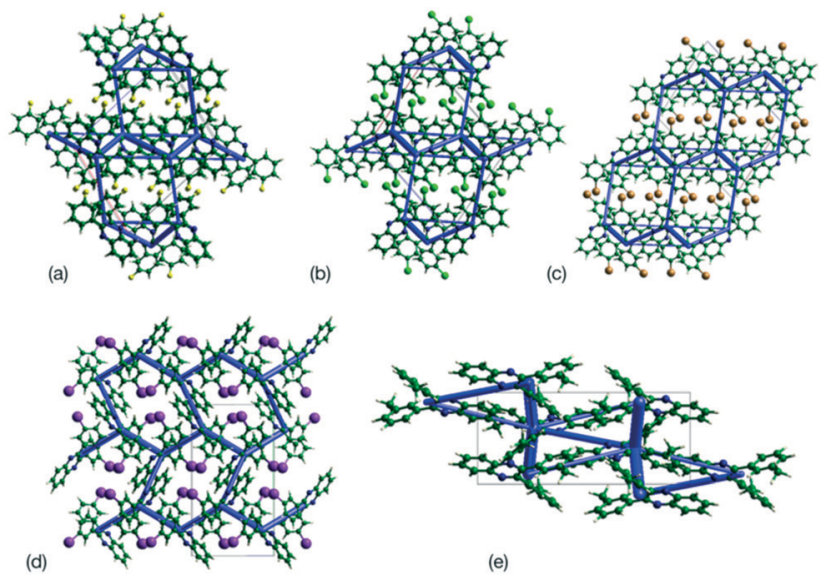

(e)

Fig. 7 Plots of the supramolecular arrangements depicting intermolecular interactions with a strength greater than $-10 \mathrm{~kJ} \mathrm{~mol}^{-1}$ in the crystal structures of (a) $Q 3,3^{\prime} F_{2}$, (b) $Q 3,3^{\prime} C_{2}$, (c) $Q 3,3^{\prime} B_{2}$, (d) $Q 3,3^{\prime} I_{2}$ and (e) $Q 3,3^{\prime} \mathrm{Me}_{2}$. Structures of $Q 3,3^{\prime} X_{2},(X=F, C l, B r)$ are viewed along the $b$-axis, while $Q 3,3^{\prime} X_{2}(X=1, M e)$ structures are viewed along the a-axis.
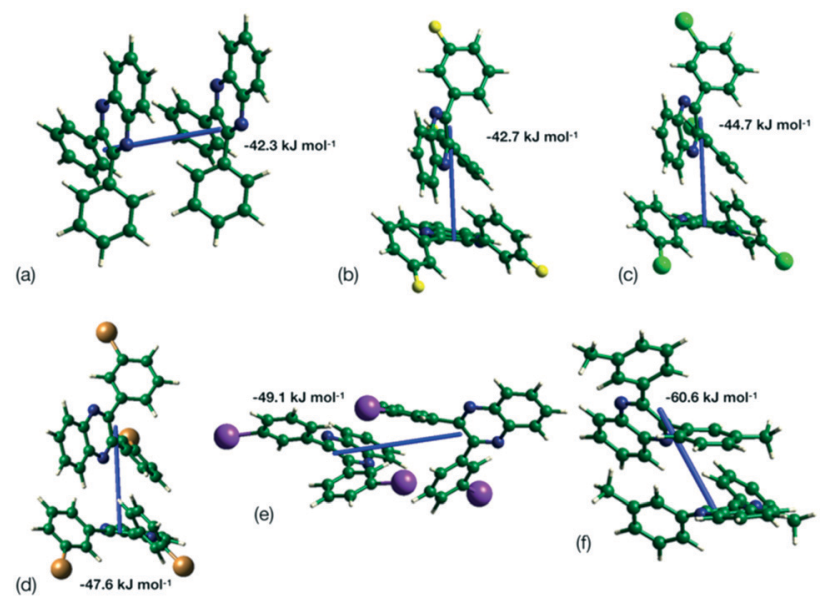

Fig. 8 Strongest interaction between molecular pairs in (a) $Q 3,3^{\prime} H_{2}$, (b) $Q 3,3^{\prime} \mathrm{F}_{2}$, (c) $\mathrm{Q} 3,3^{\prime} \mathrm{Cl}_{2}$, (d) $\mathrm{Q} 3,3^{\prime} \mathrm{Br}_{2}$, (e) $\mathrm{Q} 3,3^{\prime} \mathrm{I}_{2}$ and (f) $\mathrm{Q} 3,3^{\prime} \mathrm{Me}_{2}$.

This suggests that alternative polymorphs may be possible for the $\mathbf{Q} 3, \mathbf{3}^{\prime} \mathbf{F}_{\mathbf{2}}$ case, while the $\mathbf{Q} \mathbf{3}, \mathbf{3}^{\prime} \mathbf{I}_{\mathbf{2}}$ systems may be the lowest energy structure and favoured packing for this molecule.

\section{Polymorphism screening}

To identify any possible polymorphism, DSC data were collected on the original samples (Table 7), while the halogenated systems were recrystallised from nonpolar solvents and analysed by PXRD. These samples were identified as the same crystal forms in the cases $\mathbf{Q 3}, \mathbf{3}^{\prime} \mathbf{X}_{\mathbf{2}}(\mathrm{X}=$ F, Cl, Br, I, Fig. S3-S7†). In the case of $\mathbf{Q 3}, \mathbf{3}^{\prime} \mathbf{H}_{\mathbf{2}}$ additional peaks were noted along with the peaks for the known form, suggesting the existence of a second polymorph (Fig. S3†). The first heating cycle of the DSC analysis of $\mathbf{Q 3}, \mathbf{3}^{\prime} \mathbf{H}_{\mathbf{2}}$ (Fig. 10a) shows a melt-recrystallisation peak followed by full melting of the phase indicating a monotropic relationship between the two phases. After cooling, a second heating cycle has an exothermic recrystallisation peak leading to a phase that undergoes an endothermic phase transformation. Some of the sample stays in the original phase and undergoes a melt-recrystallisation before the remaining sample melts (Fig. 10b). These results confirm the existence of an

Table 5 Energy of strongest interaction between molecular pairs in each crystal structure

\begin{tabular}{lll}
\hline & & $\begin{array}{l}\text { Orca } \\
\text { B2PLYP-D3/def2-QZVPP/kJ } \\
\mathrm{mol}^{-1}\end{array}$ \\
\hline $\mathrm{H}$ & $\begin{array}{l}\text { CrystalExplorer HF/3-21G } \\
\text { energy/kJ mol }\end{array}$ & -43.4 \\
$\mathrm{~F}$ & -43.5 & -41.1 \\
$\mathrm{Cl}$ & -42.7 & -47.0 \\
$\mathrm{Br}$ & -44.5 & -29.4 \\
$\mathrm{I}$ & -47.5 & -53.3 \\
$\mathrm{Me}$ & -49.1 & -65.3
\end{tabular}


Table 6 Calculated lattice energies for the experimental structures

\begin{tabular}{|c|c|c|c|}
\hline $\mathrm{X}$ & COMPASSII lattice energy/kJ mol ${ }^{-1}$ & AA-CLP lattice energy/kJ mol ${ }^{-1}$ & UNI lattice energy/ $/ \mathrm{kJ} \mathrm{mol}^{-1}$ \\
\hline $\mathrm{H}$ & -126.48 & -118.16 & -141.76 \\
\hline $\mathrm{F}$ & -134.88 & -125.94 & -146.64 \\
\hline $\mathrm{Cl}$ & -152.06 & -139.80 & -169.06 \\
\hline $\mathrm{Br}$ & -155.49 & -161.64 & -159.86 \\
\hline I & -152.27 & -179.52 & -157.95 \\
\hline $\mathrm{Me}$ & -144.24 & -139.35 & -158.10 \\
\hline F in I crystal structure & -133.26 & -128.43 & -144.06 \\
\hline I in F crystal structure & -146.13 & -168.06 & -141.19 \\
\hline
\end{tabular}

additional polymorph which is more stable than the previously determined phase (Fig. 11).

DSC analysis of $\mathbf{Q} 3,3^{\prime} \mathbf{F}_{\mathbf{2}}$ and $\mathbf{Q} 3, \mathbf{3}^{\prime} \mathbf{B r}_{\mathbf{2}}$ both show melt of the given phase on the first cycle followed by recrystallisation to a lower melting phase upon heating of the cooled amorphous phase. The enthalpy and entropy of fusion rules in both cases indicates a monotropic relationship between the solid forms (Table 7). However, a second sample of Q3,3' $\mathbf{B r}_{2}$ showed reduced levels of the low temperature form (small melt recrystallisation peak), a change in the temperature of crystallisation peak and a possible glass transition in the cooling cycle that is not observed in the other sample (Fig. 12). This variation demonstrates the role of kinetics in
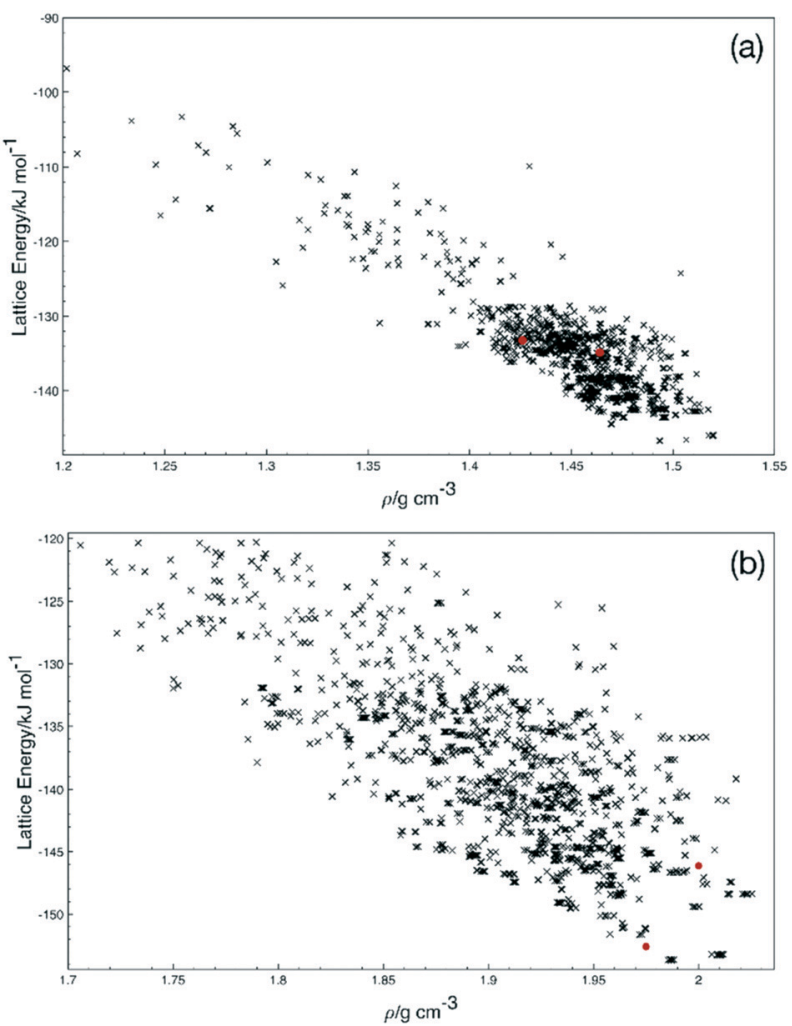

Fig. 9 Density-lattice energy plots from the crystal structure prediction for (a) $Q 3,3^{\prime} F_{2}$ and (b) $Q 3,3^{\prime} I_{2}$. Predicted structure are given as black crosses, known experimental and hypothetical structures constructed from experimental structures are red circles. the crystallisation of these phases. These kinetic factors may be directed by the synthetic route used. It has been shown that inclusion of 1-2\% of an unreacted starting material may lead to isostructural solid solutions which may be mistaken for alternative polymorphs. ${ }^{50}$ These results show that the observed isostructurality is not driven by thermodynamic factors as the stable forms are the high melting phase which have not been structurally determined. The lack of growth of alternative phases may reflect the high barriers to rotation if a different conformation is present in the stable polymorph requiring the higher temperatures for conversion. In the case of $\mathbf{Q} 3, \mathbf{3}^{\prime} \mathbf{F}_{2}$, the evidence of polymorphism supports the computational analysis where the known crystal is higher in energy than a number of predicted crystal packing and so existence of a lower energy polymorph is expected.

For $\mathbf{Q} 3, \mathbf{3}^{\prime} \mathbf{C l}_{\mathbf{2}}$ the sample shows the same melting peak on both cycles and a faster crystallisation process that occurs on the cooling cycle compared to the other samples (Fig. S8 $\dagger$ ). This compound has the highest melting point of the samples in agreement with the higher lattice energies calculated with the UNI forcefield and the dimers at B2PLYP level of theory. These energies were higher for $\mathbf{Q} 3, \mathbf{3}^{\prime} \mathbf{F}_{2}$ and $\mathbf{Q} 3, \mathbf{3}^{\prime} \mathbf{B r}_{2}$, which suggests that this crystal packing is optimal for the $\mathbf{Q} 3, \mathbf{3}^{\prime} \mathbf{C l}_{\mathbf{2}}$ and so a lack of alternative polymorphs could be expected in

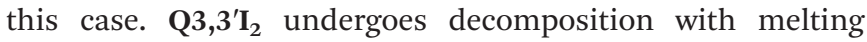
resulting in no data on the latter cycles (Fig. S9†), but the computational studies offer evidence for a lack of other polymorphs in this case.

The predicted entropies of fusion from Walden's constant $^{51}$ for fully disassociated melts is $66 \mathrm{~J} \mathrm{~K}^{-1} \mathrm{~mol}^{-1}$, indicating that the halogenated systems generally have greater levels of association. An exception is $\mathbf{Q 3}, \mathbf{3}^{\prime} \mathbf{F}_{2}$, which shows a very low entropy of fusion suggesting a low change in disorder upon melting. As the high temperature polymorph structure is unknown it is not clear what the cause is, but it may be due to increased disorder in the crystal structure.

The variability in solid form induced by the role of kinetics places limitations on the ability to generate reproducibly samples of all phases for analysis. Ongoing work will focus on obtaining suitable crystals for structural determination to confirm the conformation and packing in these phases. However, it appears that the weak hydrogen bonding, halogen bonding and van der Waals interactions in these systems are in a close balance and can be easily shifted, 
Table 7 Thermal properties of $Q 3,3^{\prime} X_{2}$

\begin{tabular}{|c|c|c|c|c|c|c|}
\hline $\mathrm{x}$ & \multicolumn{2}{|c|}{ Melting point $/{ }^{\circ} \mathrm{C}$} & \multicolumn{2}{|c|}{$\Delta_{\text {fus }} H / \mathrm{kJ} \mathrm{mol}^{-1}$} & \multicolumn{2}{|c|}{$\Delta_{\text {fus }} S / \mathrm{J} \mathrm{K}^{-1} \mathrm{~mol}^{-1}$} \\
\hline $\mathrm{H}^{a}$ & 121.5 & & 2.50 & & 6.32 & \\
\hline $\mathrm{F}$ & 138.4 & 134.8 & 13.21 & 10.50 & 32.10 & 25.72 \\
\hline $\mathrm{Cl}$ & 185.8 & 185.9 & 40.77 & 39.93 & 88.84 & 86.99 \\
\hline $\mathrm{Br}$ & 178.7 & 146.3 & 34.28 & 24.89 & 75.87 & 59.33 \\
\hline
\end{tabular}

allowing for a range of isostructural samples to be located for the smaller halogen substituents, while the greater I $\cdots$ I interactions alter the dominant motifs present in the crystal structures preventing the continuation of the isostructurality.

\section{Conclusions}

The formation of isostructural crystals for molecules through changes in substituents continues to be a challenging problem in crystal engineering. For the series of quinoxalines studied, while a set of isostructural crystals are obtained for $\mathrm{X}=\mathrm{F}, \mathrm{Cl}$ and $\mathrm{Br}$, only for $\mathrm{X}=\mathrm{Cl}$ is the thermodynamically stable polymorph grown. In the case of $\mathrm{F}$ and $\mathrm{Br}$, kinetic factors favour the growth of a metastable phase and the stable polymorph has not yet been obtained in a form

(a)

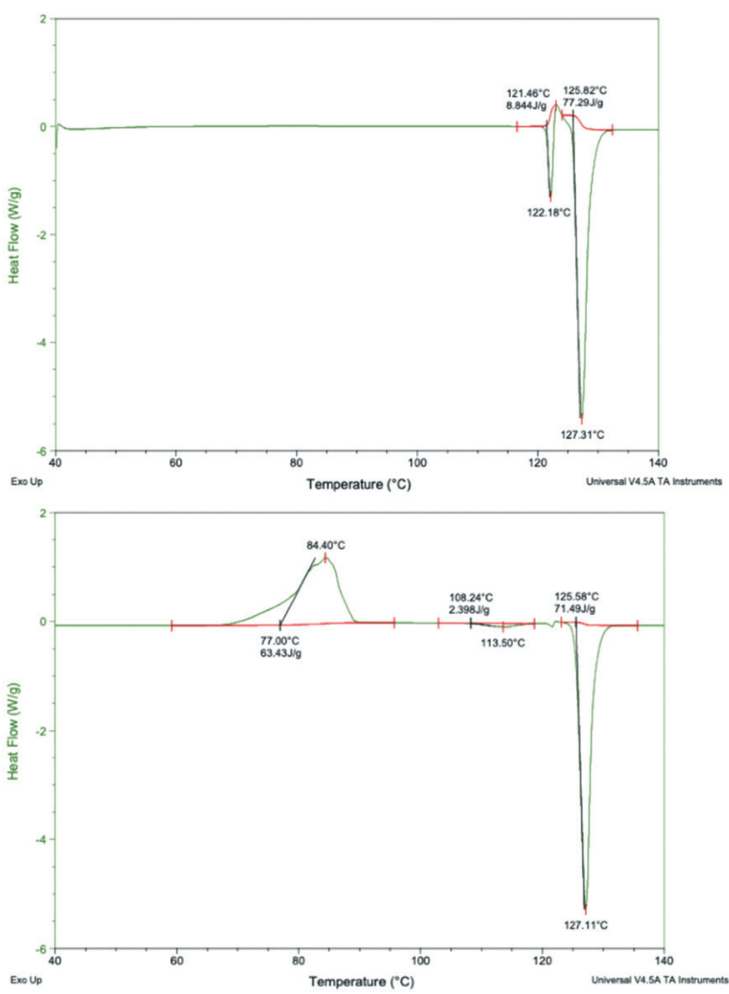

Fig. 10 DSC traces for (a) the first and (b) the second heat cycle for Q3, $3^{\prime} \mathrm{H}_{2}$. suitable for structural characterisation. Energetic calculations indicate an increase in strength of the common bonding patterns around the $\mathrm{Cl}$ system, the stability of the structural packing dropping with substitution of larger or smaller halogens. The size of the substituent does not appear to play a controlling role in the creation of isostructural crystals in this case, as replacement of $\mathrm{Cl}$ by the similarly sized $\mathrm{Me}$ group gives rise to a different conformation and packing dominated by weaker $\mathrm{CH}^{\cdots} \cdot \mathrm{N} / \mathrm{CH}^{\cdots} \cdots \pi$ hydrogen bonds. Crystal structure prediction allows for an interrogation of the energy landscape of the different polymorphs, reflecting the experimental differences in the $\mathrm{X}=\mathrm{F}$ and I systems. $\mathbf{Q} \mathbf{3}, \mathbf{3}^{\mathbf{\prime}} \mathbf{F}_{2}$ displays many potential polymorphic forms with different conformations and packing motifs, while $\mathbf{Q} 3, \mathbf{3}^{\prime} \mathbf{I}_{\mathbf{2}}$ experimental phase is one of the lowest energy crystal

(a)

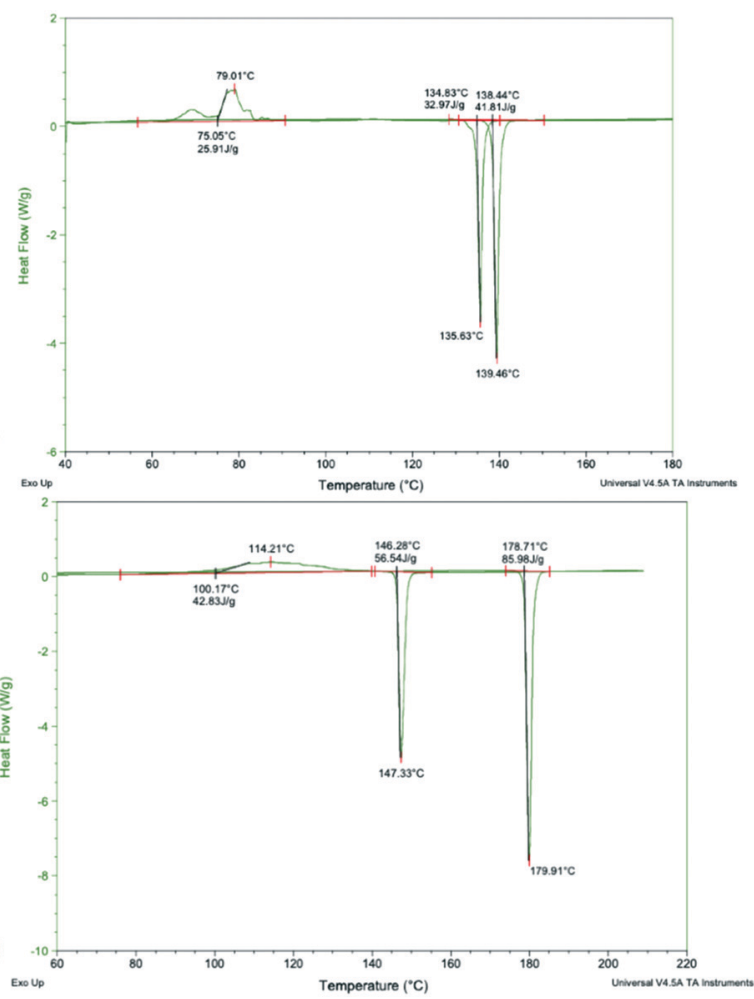

Fig. 11 First and second heating cycles for (a) $Q 3,3^{\prime} F_{2}$ and (b) $Q 3,3^{\prime}$ $\mathrm{Br}_{2}$. 


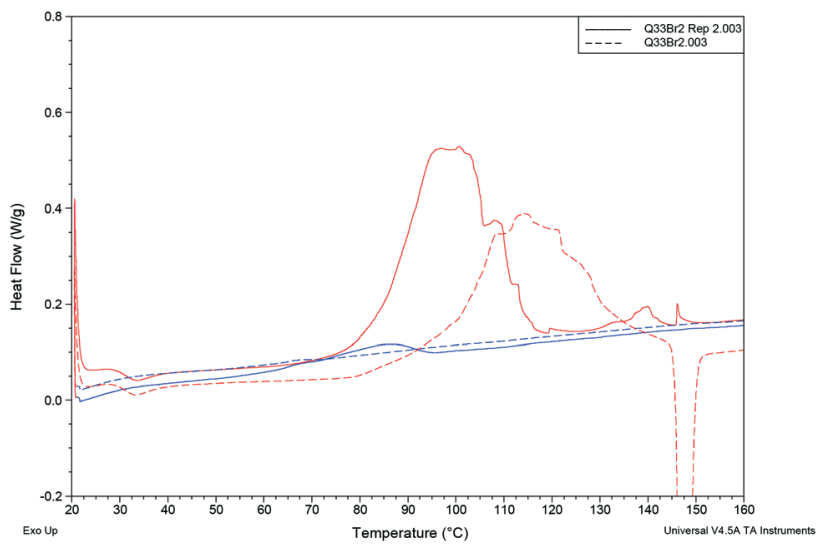

Fig. 12 Comparison of cooling cycles (blue) and heating cycles (red) for two different samples of $\mathrm{Q} 3,3^{\prime} \mathrm{Br}_{2}$.

structures and would not be expected to display a high level of polymorphism. In the case of $\mathbf{Q} 3, \mathbf{3}^{\prime} \mathbf{I}_{2}$ the introduction of potential $\mathrm{I} \cdots \mathrm{I}$ interactions alter the overall balance of forces leading to a different packing and so the isostructural set is not continued. This work shows that changes to the molecular structure can lead to isostructural crystals, but they may not be the most stable phase for a given substitution. Thus, the ability to design desired structures requires the consideration of thermodynamic and kinetic factors, along with the whole potential crystal structure landscape for even conceptually simple structures due to the balance of weak intermolecular interactions in the crystals.

\section{Author contributions}

AS: investigation, resources, methodology, validation. NWF: investigation, resources, methodology, validation. RDB: conceptualization, investigation, project administration, supervision, resources, writing - review and editing. RT: conceptualization, investigation, methodology, supervision, writing - review and editing. CCS: conceptualization, investigation, formal analysis, visualization, writing - original draft, writing - review and editing.

\section{Conflicts of interest}

There are no conflicts to declare.

\section{Notes and references}

1 G. R. Desiraju, Angew. Chem., Int. Ed., 2007, 46, 8342-8356.

2 S. R. Byrn, R. R. Pfeiffer, G. A. Stephenson, D. J. W. Grant and W. B. Gleason, Chem. Mater., 1994, 6, 1148-11158.

3 B. Rodríguez-Spong, C. P. Price, A. Jayasankar, A. J. Matzger and N. Rodriguez-Hornedo, Adv. Drug Delivery Rev., 2004, 56, 241-274.

4 S. L. Morissette, Ö. Almarsson, M. L. Peterson, J. F. Remenar, M. J. Read, A. V. Lemmo, S. Ellis, M. J. Cima and C. R. Gardner, Adv. Drug Delivery Rev., 2004, 56, 275-300.
5 G. Bolla and A. Nangia, Chem. Commun., 2016, 52, 8342-8360.

6 S. Chen and X. C. Zeng, J. Am. Chem. Soc., 2014, 136, 6428-6436.

7 A. J. Davidson, I. D. H. Oswald, D. J. Francis, A. R. Lennie, W. G. Marshall, D. I. A. Millar, C. R. Pulham, J. E. Warren and A. S. Cumming, CrystEngComm, 2008, 10, 162-165.

8 C. B. Aakeröy, T. K. Wijethunga and J. Desper, Chem. - Eur. J., 2015, 21, 11029-11037.

9 D. F. Eaton, Science, 1991, 253, 281-287.

10 J. L. Scott and K. Tanaka, Cryst. Growth Des., 2005, 5, 1209-1213.

11 A. I. Kitaigorodsky, Mixed Crystals, Springer Berlin Heidelberg, Berlin, Heidelberg, 1984, vol. 33.

12 E. Schur, E. Nauha, M. Lusi and J. Bernstein, Chem. - Eur. J., 2015, 21, 1735-1742.

13 M. Lusi, Cryst. Growth Des., 2018, 18, 3704-3712.

14 H. Lu, A. Krishna, S. M. Zakeeruddin, M. Grätzel and A. Hagfeldt, iScience, 2020, 23, 101359.

15 S. Qiu and G. Zhu, Coord. Chem. Rev., 2009, 253, 2891-2911.

16 M. Oh, L. Rajput, D. Kim, D. Moon and M. S. Lah, Inorg. Chem., 2013, 52, 3891-3899.

17 A. I. Kitaigorodsky, Molecular Crystals and Molecules, Academic Press, London, 1973.

18 C. M. Reddy, M. T. Kirchner, R. C. Gundakaram, K. A. Padmanabhan and G. R. Desiraju, Chem. - Eur. J., 2006, 12, 2222-2234.

19 A. K. S. Romasanta, D. Braga, M. T. Duarte and F. Grepioni, CrystEngComm, 2017, 19, 653-660.

20 R. Thakuria, N. K. Nath, S. Roy and A. Nangia, CrystEngComm, 2014, 16, 4681-4690.

21 P. Bombicz, N. V. May, D. Fegyverneki, A. Saranchimeg and L. Bereczki, CrystEngComm, 2020, 22, 7193-7203.

22 G. Claeson and R. Backstorm, Arkiv für Kemi, 1960, 15, 241-246.

23 M. R. Edwards, W. Jones, W. D. S. Motherwell and G. P. Shields, Mol. Cryst. Liq. Cryst., 2001, 356, 337-353.

24 M. K. Corpinot, R. Guo, D. A. Tocher, A. B. M. Buanz, S. Gaisford, S. L. Price and D.-K. Bučar, Cryst. Growth Des., 2017, 17, 827-833.

25 N. W. Thomas and G. R. Desiraju, Chem. Phys. Lett., 1984, 110, 99-102.

26 D. Cincic, T. Friščić and W. Jones, Chem. - Eur. J., 2008, 14, 747-753.

27 A. Angeloni, P. C. Crawford, A. G. Orpen, T. J. Podesta and B. J. Shore, Chem. - Eur. J., 2004, 10, 3783-3791.

28 J. A. Pereira, A. M. Pessoa, M. N. D. S. Cordeiro, R. Fernandes, C. Prudêncio, J. P. Noronha and M. Vieira, Eur. J. Med. Chem., 2015, 97, 664-672.

29 K. R. J. Thomas, M. Velusamy, J. T. Lin, C.-H. Chuen and Y.-T. Tao, Chem. Mater., 2005, 17, 1860-1866.

30 R. Sathishkumar, S. Mahapatra, T. S. Thakur and G. R. Desiraju, Curr. Sci., 2010, 99, 1807-1811.

31 É. Csikós, G. G. Ferenczy, J. G. Ángyán, Z. Böcskei, K. Simon, C. Gönczi and I. Hermecz, Eur. J. Org. Chem., 1999, 9, 2119-2125. 
32 Y. Zhan and Y. Wang, Dyes Pigm., 2020, 173, 107971.

33 A. Vogel, Textbook of Practical Organic Chemistry, ed. B. S. Furniss, A. J. Hannaford, V. Rogers, P. W. G. Smith and A. R. Tatchell, 4th edn, Longman, London, 1978, p. 916, ISBN 0-582-44350-8.

34 A. Lapworth, J. Chem. Soc., Trans., 1904, 85, 1206-1214.

35 R. Adams and C. S. Marvel, Organic Syntheses Collective Volume, 1941, 1, 94-95.

36 L. Kraszkiewicz, M. Sosnowski and L. Skulski, Tetrahedron, 2004, 60, 9113-9119.

37 N. Hayat, N. W. Fenwick, A. Saidykhan, R. Telford, W. H. C. Martin, R. T. Gallagher and R. D. Bowen, Eur. J. Mass Spectrom., 2019, 25, 457-462.

38 G. M. Sheldrick, Acta Crystallogr., Sect. A: Found. Adv., 2015, 71, 3-8.

39 G. M. Sheldrick, Acta Crystallogr., Sect. A: Found. Crystallogr., 2008, 64, 112-122.

40 O. V. Dolomanov, L. J. Bourhis, R. J. Gildea, J. A. K. Howard and H. Puschmann, J. Appl. Crystallogr., 2009, 42, 339-341.

41 F. Neese, WIREs Comput. Mol. Sci., 2012, 2, 73-78.

42 S. Grimme, J. G. Brandenburg, C. Bannwarth and A. Hansen, J. Chem. Phys., 2015, 143, 054107.

43 A. Gavezzotti, New J. Chem., 2011, 35, 1360-1368.
44 A. Gavezzotti, Molecular Aggregation, Oxford University Press, 2007.

45 J. A. Nelder and R. Mead, Comput J, 1965, 7, 308-313.

46 M. J. Turner, J. J. McKinnon, S. K. Wolff, D. J. Grimwood, P. R. Spackman, D. Jayatilaka and M. A. Spackman, CrystalExplorer, University of Western Australia, 2017.

47 S. Grimme, S. Ehrlich and L. Goerigk, J. Comput. Chem., 2011, 32, 1456-1465; S. Grimme, J. Antony, S. Ehrlich and H. Krieg, J. Chem. Phys., 2010, 132, 154104; F. Weigend and R. Ahlrichs, Phys. Chem. Chem. Phys., 2005, 7, 3297; F. Weigend, Phys. Chem. Chem. Phys., 2006, 8, 1057; A. Hellweg, C. Hattig, S. Hofener and W. Klopper, Theor. Chem. Acc., 2007, 117, 587; J. Chmela and M. E. Harding, Mol. Phys., 2018, 116, 1523.

48 Y. Mo, Wiley Interdiscip. Rev. Comput. Mol. Sci., 2011, 1, 164-171.

49 M. Tremayne, L. Grice, J. C. Pyatt, C. C. Seaton, B. M. Kariuki, H. H. Y. Tsui, S. L. Price and J. C. Cherryman, J. Am. Chem. Soc., 2004, 126, 7071-7081; Z. Zhu, Z. Xu and W. Zhu, J. Chem. Inf. Model., 2020, 60, 2683-2696.

50 A. Poulain, M. Kubicki and C. Lecomte, Acta Crystallogr., Sect. B: Struct. Sci., Cryst. Eng. Mater., 2014, 70, 973-982.

51 S. N. Black, C. L. Woon and R. J. Davey, Cryst. Growth Des., 2018, 18, 6918-6928. 